\title{
Clinicopathologic Features of Ovarian Mixed Mesodermal Tumors and Carcinosarcomas
}

\author{
Keith Y. Terada, M.D., Terri L. Johnson, M.D., Michael Hopkins, M.D., and James A. Roberts, M.D. \\ Department of Obstetrics and Gynecology and Department of Pathology, University of Michigan School of Medicine
}

Received July 20, 1987

Over a 27 -year period there were 15 patients treated at the University of Michigan Medical center for carcinosarcomas or mixed mesodermal tumors of the ovary. Overall median survival was 11 months. Median survival was 4 months in patients with carcinosarcomas and 13 months in patients with mixed mesodermal tumors $(P=0.066)$. Patients with a chondrosarcomatous element had a significantly longer median survival than patients with mixed mesodermal tumors lacking cartilaginous differentiation $(P=0.031)$. The presence of a rhabdomyosarcomatous element did not affect survival. The size of the primary tumor, presence of ascites, FIGO stage, and degree of initial resection (optimal versus suboptimal) did not affect survival. There was no difference in survival between patients receiving postoperative chemotherapy and patients undergoing postoperative irradiation. 1989 Academic Press, Inc.

\section{INTRODUCTION}

Malignant mixed mesodermal tumors and carcinosarcomas of the ovary are unusual and constitute less than $1 \%$ of all ovarian malignancies [1].

These tumors are composed of intimately admixed carcinomatous and sarcomatous components. Tumors containing a "heterologous" element, i.e., chondrosarcoma or rhabdomyosarcoma, are termed mixed mesodermal tumors; if heterologous differentiation is absent the ovarian tumor is termed carcinosarcoma. These tumors have been noted to be aggressive and rapidly progressive; the only effective therapy has been surgical resection of localized disease $[2,3]$. The majority of patients, however, present with tumor extending beyond the ovary and require additional treatment. The present study was undertaken to review the clinicopathologic features, treatment, and outcome of patients with mixed mesodermal tumors and carcinosarcomas of the ovary.

\section{MATERIALS AND METHODS}

The medical records of all patients presenting to the University of Michigan Medical Center with carcinosar- comas or mixed mesodermal tumors of the ovary from 1959 to 1986 were retrospectively reviewed. Clinical features, surgical findings, treatment, and follow-up were all reviewed in detail. All histologic materials were reviewed by one of the primary authors (T.L.J.). Four patients who had been previously reported [3], however, were included in the present report to further analyze treatment outcome and histopathology. Tissue blocks were recut for special stains, including periodic acid-Schiff (PAS) and $\alpha$-1-antitrypsin.

Survival was calculated using the product-limit method of Kaplan and Meier [4]. Gehan's generalized Wilcoxon test was used to compare survival distributions [5].

\section{RESULTS}

There were 15 patients treated during this period for carcinosarcomas or mixed mesodermal tumors of the ovary. Patients were all white with a mean age of 63 years (range $44-72$ years). Mean parity was 3.1 with $13 \%$ of patients nulliparous. One patient had undergone prior hysterectomy for a benign condition and 1 patient had undergone prior radiotherapy for a squamous cell carcinoma of the cervix. One patient had concomitant endometriosis, and 1 patient had a simultaneous well-differentiated adenocarcinoma of the endometrium.

The majority of patients presented with nonspecific symptoms characteristic of ovarian tumors: Increased abdominal girth, bloating, fatigue, and lower abdominal discomfort. One patient was asymptomatic at the time of presentation.

All patients underwent an initial laparotomy. Operative findings revealed ovarian tumors from 3 to $25 \mathrm{~cm}$ in diameter; tumor size averaged $15 \mathrm{~cm}$. Seven cases involved both ovaries, six involved the right ovary only and two arose in the left ovary. The neoplasms were usually solid and cystic with areas of hemorrhage and necrosis. Solid areas often had a fleshy consistency and were variegated in appearance. Nine patients had ascites, including 2 
patients with bloody ascites. All patients had tumor extending beyond the ovary; 4 patients were FIGO stage II and 11 patients were FIGO stage III. Sites of metastases involved the omentum, peritoneum, serosal surfaces, and pelvic soft tissue. In 5 patients an optimal cytoreductive operation was accomplished (residual tumor less than $1.5 \mathrm{~cm}$ in greatest diameter). Ten patients were left with suboptimal disease.

Following initial surgery, one patient had no further treatment. Five patients underwent pelvic irradiation and one patient underwent abdominopelvic irradiation. Eight patients received chemotherapy. Chemotherapeutic regimens varied considerably; however, they consisted of either a single alkylating agent (melphalan or chlorambucil) or combinations utilizing vincristine, actinomycin D, cyclophosphamide, Adriamycin, cis-platinum, methotrexate, and/or dacarbazine. The most frequently used combination was vincristine, actinomycin $\mathrm{D}$, and cyclophosphamide.

Neoplasms were classified as mixed mesodermal tumors if they contained both a carcinomatous component and a sarcomatous component with foci of chondrosarcoma, rhabdomyosarcoma, osteosarcoma, and/or liposarcoma. Carcinosarcomas contained a malignant epithelial com- ponent and a sarcomatous component without differentiation toward cartilage, skeletal muscle, bone, or adipose tissue.

In all of the ovarian tumors, the malignant epithelial component was moderately to poorly differentiated adenocarcinoma. The proportion of carcinoma present in the tumors ranged from $10 \%$ to $95 \%$. Ten of the tumors $(67 \%)$ had a predominantly papillary adenocarcinomatous component while in five cases (33\%) the adenocarcinoma had a predominantly glandular growth pattern which resembled endometrioid carcinoma. All of the neoplasms had areas of adenocarcinoma with a solid growth pattern. Generally, the pattern of adenocarcinoma present in the primary tumor was also present in metastatic and recurrent tumors, although in several cases the degree of differentiation decreased and the proportion of solid growth pattern was greater in the metastatic tumors. In one patient, metastases had an adenosquamous component (Fig. 1) while the primary ovarian tumor had a pure adenocarcinoma component with a glandular growth pattern.

Eleven tumors contained areas of chondrosarcoma or rhabdomyosarcoma and were therefore classified as mixed

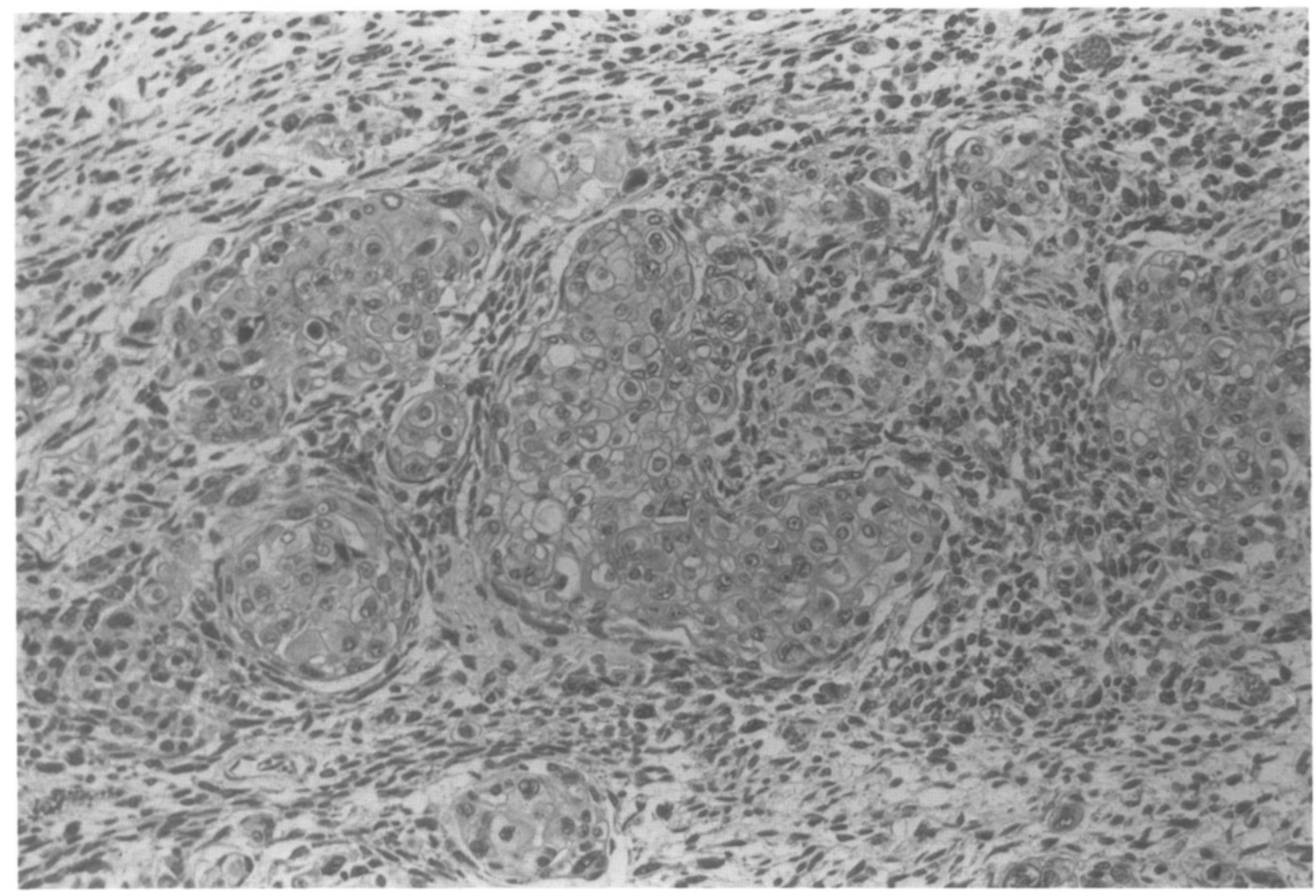

FIG. 1. Foci of adenosquamous carcinoma in area of undifferentiated sarcoma. $\mathrm{H} \& \mathrm{E}, \times 16$. 


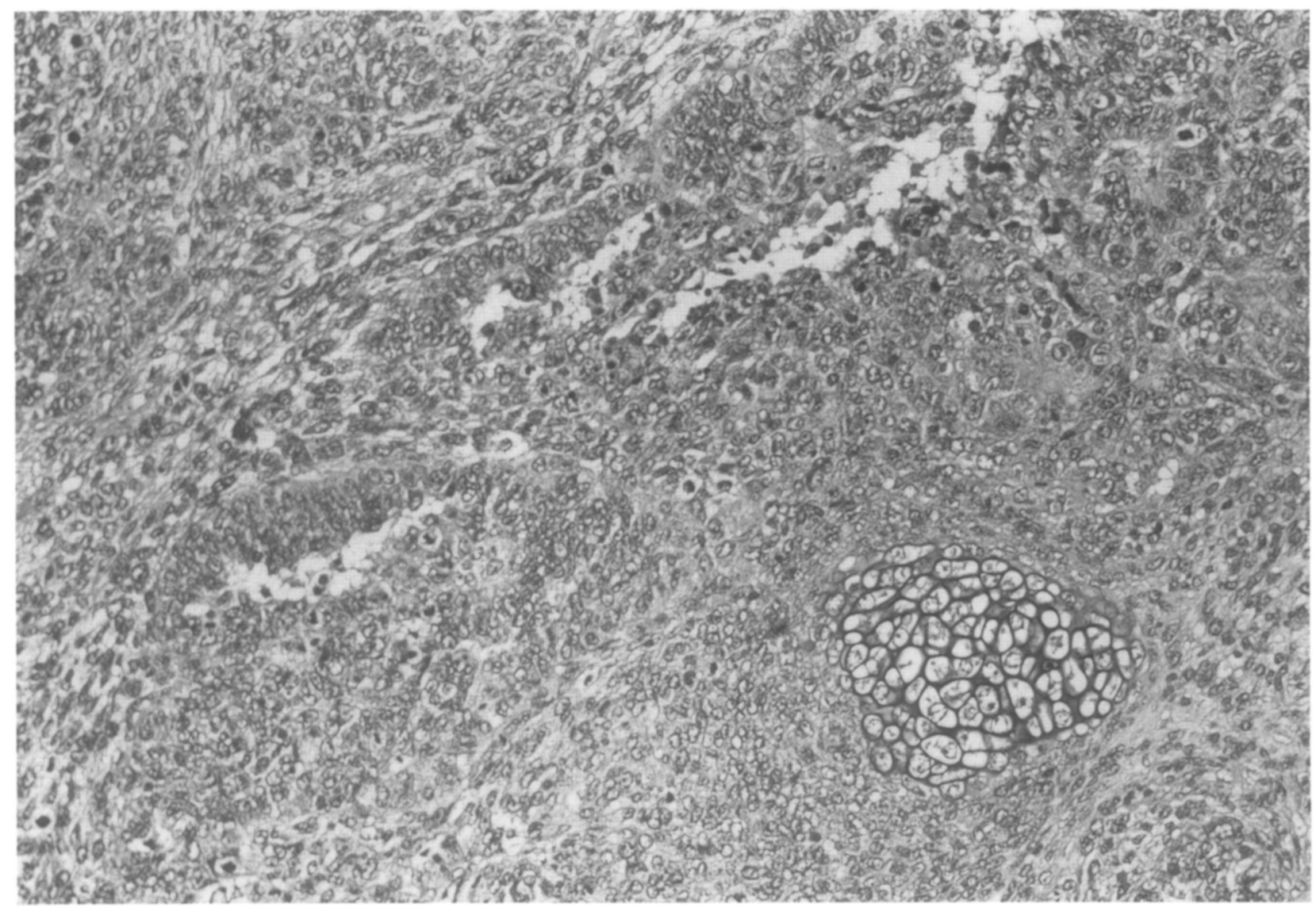

FIG. 2. Glandular adenocarcinoma (left and mid-upper) with focus of chondrosarcoma (right lower) in area of undifferentiated sarcoma. H \& $\mathrm{E}, \times 10$.

mesodermal tumors of ovary (Fig. 2). There were no foci of osteosarcoma or liposarcoma in any of the neoplasms. Six tumors contained chondrosarcoma, 2 contained rhabdomyosarcoma, and 3 contained both components. The foci of rhabdomyosarcoma were identified in $\mathrm{H} \& \mathrm{E}$ stained slides as large eosinophilic polygonal or strapshaped cells with large eccentric nuclei and prominent nucleoli. Immunoperoxidase stains for desmin and myoglobin were done to confirm the presence of rhabdomyoblasts. In all of the mixed mesodermal tumors, the areas of chondrosarcoma and rhabdomyosarcoma accounted for less than $50 \%$ of the sarcomatous component. Most of the sarcomatous areas were composed of undifferentiated and myxoid sarcoma, similar to the nonepithelial component in the four carcinosarcomas. The areas of undifferentiated sarcoma in both the mixed mesodermal tumors and the carcinosarcomas contained anaplastic spindle-cells with a very high mitotic rate including abnormal forms and many bizzare, pleomorphic tumor giant cells. The myxoid sarcoma areas were less cellular than the undifferentiated sarcoma and were composed of spindle cells in a basophilic myxohyaline background
(Fig. 3). Mitotic figures and giant cells were less numerous in the areas of myxoid sarcoma. PAS-positive, diastaseresistant hyaline droplets which stained with $\alpha$-antitrypsin were present in the sarcomatous areas of $80 \%$ of the tumors.

Median overall survival was 11 months and was not affected by stage, optimal versus suboptimal initial resection, size of the primary tumor, or the presence of ascites. The mean progression free interval was 8 months and the mean survival following recurrence was 3 months. There was no significant difference in survival between patients receiving chemotherapy and patients undergoing postoperative irradiation. In addition, there was no chemotherapeutic agent or regimen that appeared more efficacious than the others.

Median survival was 4 months in patients with carcinosarcomas and 13 months in patients with mixed mesodermal tumors. This difference was of borderline statistical significance $(P=0.066)$. Patients with a chondrosarcomatous element had a significantly longer median survival (13 months) than patients with mixed mesodermal tumors lacking cartilaginous differentiation 


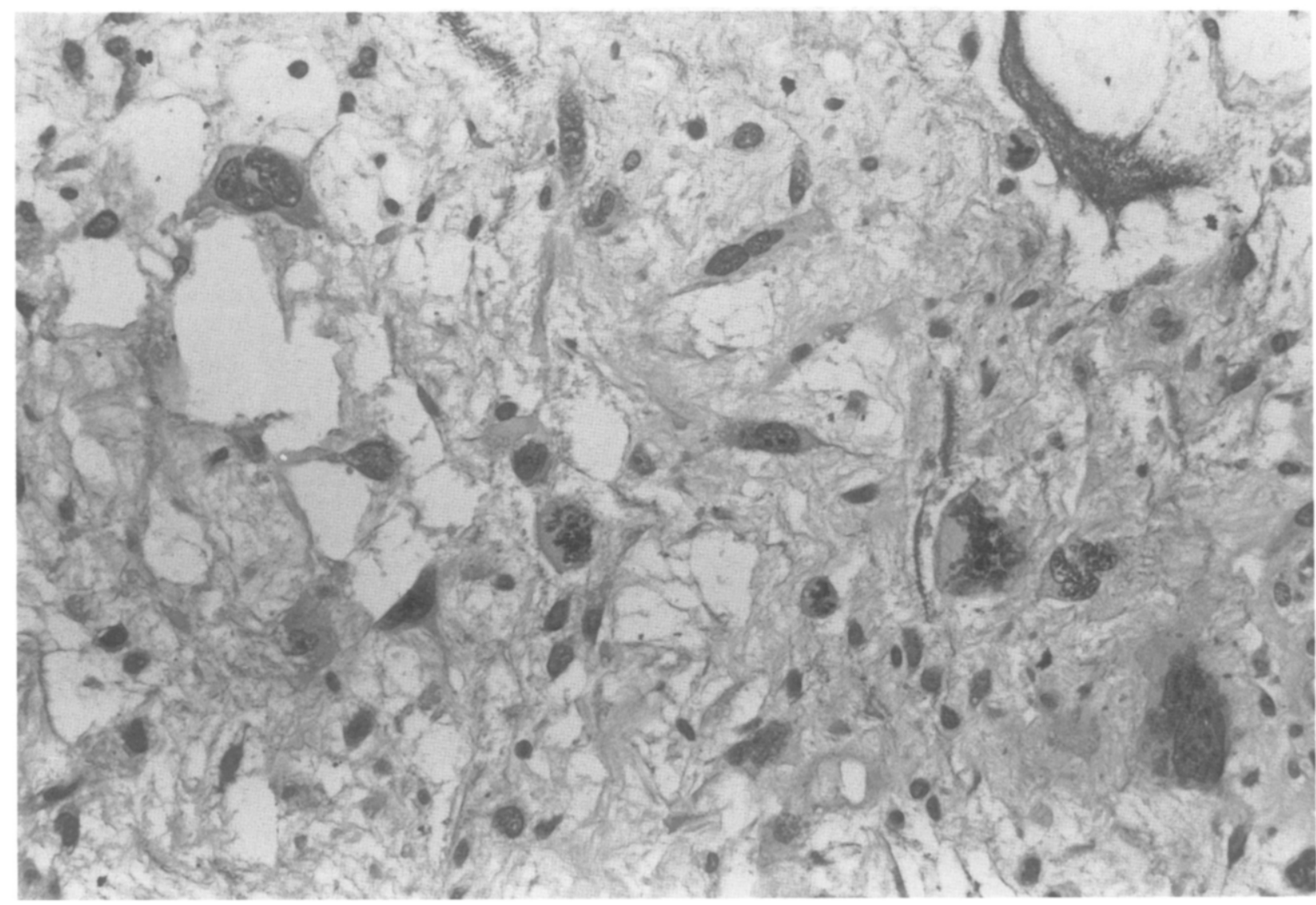

FIG. 3. Myxoid sarcoma with pleomorphic tumor giant cells. H \& E, $\times 25$.

(6 months, $P=0.031$ ). The presence or absence of a rhabdomyosarcomatous element did not significantly affect survival.

Patients developing recurrent disease presented with a mass in the abdomen and/or pelvis (11 patients) or ascites ( 3 patients). All patients with recurrence died of disease. Five patients underwent postmortem examination. Two patients had both carcinomatous elements and sarcomatous elements present. Three patients had only sarcomatous elements identified microscopically at postmortem examination. All 5 patients had tumor confined to the abdomen and pelvis and had tumor extensively involving the intraabdominal organs. Sites of metastases are noted in Table 1.

\section{DISCUSSION}

Mixed mesodermal tumors and carcinosarcomas of the ovary are unusual tumors, with less than 300 cases reported in the literature $[2,6]$. This study confirms previous reports that these malignancies are rapidly progressive and almost uniformly fatal [7-10]. Clinically, presenting symptoms and physical signs are characteristic of ovarian malig- nancies, i.e, bloating, fatigue, increased abdominal girth, etc. Although nulliparity and endometriosis have both been associated with these malignancies, endometriosis was noted in only one patient and nulliparity in two patients in the present series. One patient in the series had undergone prior pelvic radiotherapy. A review of the literature indicates that endometriosis and/or prior radiotherapy are probably not significant etiologic factors in this disease $[2,11]$.

TABLE 1

Sites of Metastases at Postmortem Examination, Five Patients

\begin{tabular}{lc}
\hline \multicolumn{1}{c}{ Sites } & Number of patients \\
\cline { 2 - 2 } Peritoneal, serosal surfaces & 5 \\
Liver & 3 \\
Diaphragm & 3 \\
Periaortic lymph nodes & 4 \\
Pelvic soft tissue & 3 \\
Omentum & 2 \\
Spleen & 1 \\
Pancreas & 1 \\
Kidney & 1 \\
\hline
\end{tabular}


Operative findings and patterns of metastases generally resemble that found with epithelial ovarian carcinomas. Metastases most often involve the peritoneal and serosal surfaces and pelvic soft tissues. None of the patients had gross involvement of the retroperitoneal lymph nodes at the time of initial laparotomy; however, these were not routinely biopsied. Retroperitoneal lymph nodes were a common site of metastases at postmortem examination. Therefore, a careful and complete initial staging procedure is recommended as for patients with epithelial malignancies.

In the present study stage did not appear to affect survival. All patients, however, had tumor extension beyond the ovary. Previous reports indicate that patients presenting with earlier stages may have a slightly better prognosis [7,10]; and surgical resection of tumor limited to one ovary appears to represent the most realistic chance of long-term survival $[3,10]$.

Fourteen of 15 patients in this series received additional treatment following surgery. As with previous studies there was no chemotherapeutic agent or regimen that appeared clearly superior [12]. In addition, survival with postoperative radiotherapy was not significantly different than with chemotherapy. Other reports indicate that postoperative treatment with combined radiotherapychemotherapy may occasionally be effective in achieving long-term remission $[13,14]$.

The most significant histopathologic variable in the present study was the presence of a chondrosarcomatous component in patients with mixed mesodermal tumors. These patients had a significantly longer median survival than patients without cartilaginous differentiation. There was no significant difference in survival between patients with carcinosarcomas and mixed mesodermal tumors. Dehner et al. reported a longer median survival in patients with carcinosarcomas than patients with mixed mesodermal tumors; their population, however, contained a greater proportion of early stage lesions in patients with carcinosarcomas [8]. Stage and stromal type appear to be interrelated prognostic determinants $[8,10]$; with advanced stage disease there may not be a significant difference in survival.

The presence of PAS-positive, diastase-resistant hyaline droplets staining positive for $\alpha$-antitrypsin was noted in a majority of the tumors. Although this has been noted previously, the clinical significance remains unclear [15]. The possibility of utilizing serum levels of $\alpha$-antitrypsin as a tumor marker should be investigated in the future.

\section{REFERENCES}

1. Azoury, R. S., and Woodruff, J. D. Primary ovarian sarcomas, Obstet. Gynecol. 37, 920 (1971).

2. Hanjani, P., Peterson, O., Lipton, S. E., and Nolte, S. A. Malignant mixed mesodermal tumors and carcinosarcoma of the ovary: Report of eight cases and review of the literature, Obstet. Gynecol. Surv. 38, 537 (1983).

3. Fenn, M. E., and Abell, M. R. Carcinosarcoma of the ovary, Amer. J. Obstet. Gynecol. 110, 1066 (1971).

4. Kaplan, E. L., and Meier, P. Nonparametric estimation from incomplete observations, J. Amer. Stat. Assoc. 53, 203 (1965).

5. Gehan, E. A. A generalized Wilcoxon test for comparing arbitrarily singly-censored samples, Biometrika 52, 203 (1965).

6. Anderson, B., Turner, D. A., and Benda, J. Ovarian sarcoma, Gynecol. Oncol. 26, 183 (1987).

7. Morrow, C. P., d'Ablaing, G., Brady, L. W., Blessing, J. A., and Hreshchyshyn, M. M. A clinical and pathologic study of 30 cases of malignant mixed mullerian epithelial and mesenchymal ovarian tumors: A gynecologic oncology group study, Gynecol. Oncol. 18, 278 (1984)

8. Dehner, L. P., Norris, H. J., and Taylor, H. B. Carcinosarcomas and mixed mesodermal tumors of the ovary, Cancer 27, 207 (1971).

9. Barwick, K. W., and LiVolsi, V. A. Malignant mixed mesodermal tumors of the ovary, a clinicopathologic assessment of 12 cases, Amer. J. Surg. Pathol. 4, 37 (1980).

10. Dictor, M. Malignant mixed mesodermal tumor of the ovary: A report of 22 cases, Obstet. Gynecol. 65, 720 (1985).

11. Hernandez, W., DiSaia, P. J., Morrow, C. P., and Townsend, D. E. Mixed mesodermal sarcoma of the ovary, Obstet. Gynecol. 49(Suppl. 1), 59 (1977).

12. Lele, S. B., Piver, M. S., and Barlow, J. J. Chemotherapy in management of mixed mesodermal tumors of the ovary, Gynecol. Oncol. 10, 298 (1980).

13. Carlson, J. A., and Day, T. G. Five-year survival following combination radiotherapy and chemotherapy for recurrent mixed mesodermal sarcoma of the ovary, Gynecol. Oncol. 22, 129 (1985).

14. Carlson, J. A., Edwards, C., Wharton, J. T., Gallager, H. S., Delclos, L., and Rutledge, F. Mixed mesodermal sarcoma of the ovary, treatment with combination radiotherapy and chemotherapy, Cancer 52, 1473 (1983).

15. Dictor, M. Ovarian malignant mixed mesodermal tumor: The occurrence of hyaline droplets containing alpha-1-antitrypsin, Hum. Pathol. 13, 930 (1982). 